\title{
Article \\ Effect of Voids and Internal State Variables in Elasticity of Porous Bodies with Dipolar Structure
}

\author{
Marin Marin ${ }^{1, *(\mathbb{D}}$, Sorin Vlase ${ }^{2} \mathbb{D}$, Iana Fudulu ${ }^{1}$ and Georgiana Precup ${ }^{1}$ \\ 1 Department of Mathematics and Computer Science, Transilvania University of Brasov, \\ 500036 Brasov, Romania; im.fudulu@unitbv.ro (I.F.); g.precup@unitbv.ro (G.P.) \\ 2 Department of Mecanical Engineering, Transilvania University of Brasov, 500036 Brasov, Romania; \\ svlase@unitbv.ro \\ * Correspondence: m.marin@unitbv.ro
}

Citation: Marin, M.; Vlase, S.;

Fudulu, I.; Precup, G. Effect of Voids and Internal State Variables in Elasticity of Porous Bodies with Dipolar Structure. Mathematics 2021, 9, 2741. https://doi.org/10.3390/ math9212741

Academic Editor: Efstratios Tzirtzilakis

Received: 23 August 2021

Accepted: 27 October 2021

Published: 28 October 2021

Publisher's Note: MDPI stays neutral with regard to jurisdictional claims in published maps and institutional affiliations.

\begin{abstract}
In this study, we will prove that the presence of the voids and of the internal state variables in an elastic body with dipolar structure have no effect on the result of uniqueness regarding the solution of the initial-boundary value problem from this context. First, we put down the basic equations and conditions which define the initial-boundary value problem in the context. Then, by means of three theorems, we prove some auxiliary estimates that underlie the result of uniqueness. Finally, by means of these estimates and by using the Gronwall's inequality, we prove our main result.
\end{abstract}

Keywords: elastic body; dipolar structure; internal state variables; uniqueness; Gronwall's inequality

\section{Introduction}

First, we want to argue the motivation for considering the voids of material.

In recent years, there has been a significant increase of the number of studies dedicated to the theory of porous media.

This is why we have included our present study in the elasticity of media with voids or vacuous pores. It is considered that the initiator in this theory was the study [1].

As we know (see also in [2,3]) in that theory a new degree of freedom appears for a more complete description of the behavior of materials regarding the mechanical properties. The importance of porous bodies it is seen in geophysics context (geological materials, for example, rocks or soils) and in the case of granular materials (artificially obtained).

Of course, first studies were elaborated in the linear case, without taking into account the thermal effect (see, for instance, in [2]). Then, the studies approached the thermoelasticity of bodies with vacuous pores (see in [4]). Another papers in this topic can be found in $[5,6]$. Furthermore, in our paper we consider a dipolar structure, as a particular case of the microstructure, which was introduced by Eringen. For this we can enumerate the papers $[7,8]$. After that, many works appeared on microstructure media which increased the importance of this theory.

The dipolar structure, as a particular case of the microstructure, is intended to eliminate, at least in part, some contradictions in the theory of classical elasticity (one, very well known, the heat waves propagate at an infinite speed). Some outstanding researchers approached this kind of structure. We enumerate some of them, namely, the papers [9-11], that are conclusive for this structure. Different features of the generalized theories are approached in [12-22].

Now, we want to argue the motivation for considering the internal state variables.

Lately, the number of studies dedicated to theory of bodies with internal state variables has increased. The internal state variables can be considered as a means to evaluate mechanical properties of a body, but also many other effects, like electric, magnetic, chemical, and so on. 
For the first time, the internal state variables were considered to describe the evolution of the viscoelastic bodies in the theory of thermoelasticity (see, for instance, Chirita [23]). Then, it was found that these variables are useful for studying behavior and other types of materials.

Therefore, in the study Nachlinger and Nunziato [24] the internal state variables are used for evolution of finite deformations without conduction of heat, for an onedimensional body.

The authors of [25] describe implications of so-called Bammann internal state variable with an good benefit to geological materials, like silicate rocks and other.

In [26], it is shown that the capillary hysteresis can be described with the help of a set of variables of internal state type. In [27], we find a model for an amorphous thermoplastics by using a set of variables of internal state type which physically motivates this thermodynamic approach.

In [28], the authors develop an elastic-viscoplastic theory for the behavior deformation of polymeric and metallic glasses. A state variable based gradient theory is presented in [29], and this theory provides a consistent framework and strong coupling in order to prescribe the dissipation and energy storage. For some results on anti-plane states in an anisotropic elastic body and for orthotropic or isotropic plates, we suggest $[30,31]$.

\section{Main Equations and Conditions}

We will consider $D$ be an open domain of the Euclidean space $R^{3}$ which is occupied, at the initial moment $t=0$, by an elastic body with internal state variables and dipolar structure. The surface $\partial D$ is the border of the domain $D$ and is a closed and bounded set which permits the application of the theorem of divergence. A point in $D$ is represented in the form $\left(x_{i}\right)$ or $(x)$. For the time variable $t$ we suppose that $t \in\left[0, t_{0}\right)$.

The derivative of a function $f$ regarding to a spatial argument $x_{j}$ is denoted by $f, i$ and $\dot{f}$ is the derivative of $f$ regarding the time variable $t$.

To characterize the evolution of an elastic dipolar body with voids there are used the kinematic variables that follow:

$$
v_{m}=v_{m}(x, t), \phi_{j k}=\phi_{j k}(x, t), \sigma=\sigma(x, t),(x, t) \in D \times\left[0, t_{0}\right),
$$

where $v_{m}$ are the components of the displacement field and $\phi_{j k}$ - the components of the dipolar displacement field and $\sigma$ is the volume distribution function.

In our following considerations, we will use $\varphi$ as the volume distribution function, where $\varphi=\sigma-\sigma_{0}$, where $\sigma_{0}$ is the value of $\sigma$ in the initial state.

By using the Green and Rivlin's procedure, we can consider another deformation which differ from the given deformation only by a superposition of a rigid motion which consists of a rotation having a constant angular velocity. We must assume that for this motion, all other properties of the media remain unaffected by this superposition. As a consequence, we obtain the next kinematic relations, that provides expressions the measures of the strain, namely, $e_{i j}, \varepsilon_{i j}$ and $\gamma_{i j k}$, with regard to the variables of motion:

$$
\begin{array}{r}
e_{i j}=\frac{1}{2}\left(u_{j, i}+u_{i, j}\right), \varepsilon_{i j}=u_{j, i}-\phi_{i j}, \\
\gamma_{i j k}=\phi_{j k, i} .
\end{array}
$$

We will denote the internal state variables by $\xi_{v}, v=1,2, \ldots, n$, and we use the notation $\xi_{v}^{0}$ for the values of the variables in the initial state of the body. Being in the context of a linear theory it is natural to use as internal variables the difference of the above, $\omega_{v}$, that is

$$
\omega_{v}=\xi_{v}-\xi_{v}^{0} .
$$


We will make considerations only in the particular case in which the solids have a point which is the center of symmetry. Furthermore, we consider that for the body, which in its initial state, has no stress, is free from intrinsic equilibrated forces and has no body couples, the internal energy density is a form of quadratic power with respect to its constitutive independent variables. Thus, by using the principle of energy conservation, we obtain the following expression of the density of internal energy:

$$
\begin{array}{r}
\Psi=\frac{1}{2} A_{i j m n} e_{i j} e_{m n}+G_{i j m n} e_{i j} \varepsilon_{m n}+F_{i j m n r} e_{i j} \gamma_{m n r}+\frac{1}{2} B_{i j m n} \varepsilon_{i j} \varepsilon_{m n} \\
+D_{i j m n r} \varepsilon_{i j} \gamma_{m n r}+\frac{1}{2} C_{i j k m n r} \gamma_{i j k} \gamma_{m n r}+a_{i j k} e_{i j} \varphi_{, k}+b_{i j k} \varepsilon_{i j} \varphi_{, k}+c_{i j k m} \gamma_{i j k} \varphi_{, k}+ \\
\frac{1}{2} p_{m n} \varphi_{, m} \varphi_{, n}+\alpha_{i j v} e_{i j} \omega_{v}+\beta_{i j v} \varepsilon_{i j} \omega_{v}+\delta_{i j k v} \gamma_{i j k} \omega_{v}+f_{i v} \varphi_{, i} \omega_{v} .
\end{array}
$$

The above coefficients $A_{i j m n}, B_{i j m n}, \ldots, a_{i j k}, \ldots, f_{i v}$ characterize the elastic properties of a body with pores and with internal state variable and are called the constitutive coefficients. In general, these coefficients depend on the point $x$ and in the particular case when the body is homogeneous, they have constant value.

With the help of this density of internal energy, we can obtain the constitutive relations that follow, which provide the expressions for the measures of the stress, $t_{i j}, \tau_{i j}, m_{i j k}, h_{i}$, as functions depending on the tensors of strain:

$$
\begin{array}{r}
t_{i j}=\frac{\partial \Psi}{\partial e_{i j}}=A_{i j m n} e_{m n}+G_{m n i j} \varepsilon_{m n}+F_{m n r i j} \gamma_{m n r}+a_{i j k} \varphi_{, k}+\alpha_{i j v} \omega_{v}, \\
\tau_{i j}=\frac{\partial \Psi}{\partial \varepsilon_{i j}}=G_{i j m n} e_{m n}+B_{i j m n} \varepsilon_{m n}+D_{i j m n r} \gamma_{m n r}+b_{i j k} \varphi_{, k}+\beta_{i j v} \omega_{v}, \\
m_{i j k}=\frac{\partial \Psi}{\partial \gamma_{i j k}}=F_{i j k m n} e_{m n}+D_{m n i j k} \varepsilon_{m n}+C_{m n r i j k} \gamma_{m n r}+c_{i j k m} \varphi_{, m}+\delta_{i j k v} \omega_{v}, \\
h_{i}=\frac{\partial \Psi}{\partial \varphi_{, i}}=a_{i j k} e_{j k}+b_{i j k} \varepsilon_{j k}+c_{i j s m} \gamma_{j s m}+f_{i v} \omega_{v} .
\end{array}
$$

The procedure used by Green and Rivlin in the case of classical elasticity, can be also used to obtain the balance laws in the context of elasticty of porous bodies with dipolar structure, namely,

- $\quad$ the equations of motion:

$$
\begin{gathered}
\left(t_{m n}+\tau_{m n}\right)_{, n}+\rho f_{m}=\rho \ddot{v}_{m}, \\
m_{i j k, i}+\tau_{j k}+\rho g_{j k}=I_{k r} \ddot{\phi}_{j r} ;
\end{gathered}
$$

- the balance of the equilibrated forces:

$$
h_{i, i}+\rho l=\rho k \ddot{\varphi} .
$$

In the context of a linear approximation, we can use a suggestion from [1] so that the inequality of the entropy production leads to the following equation:

$$
\dot{\omega}_{v}=f_{v}
$$

where

$$
f_{v}=g_{i j v} e_{i j}+h_{i j v} \varepsilon_{i j}+l_{i j k v} \gamma_{i j k}+q_{v \beta} \omega_{\beta} .
$$

The significance of other notations used in the previous relations is the following:

- $\quad \rho$ - the constant mass density;

- $\quad t_{i j}, \tau_{i j}, m_{i j k}$ - the tensors of the stress; 
- $\quad I_{i j}$-the tensor of the inertia;

- $\quad k$-microinertia;

- $\quad f_{m}$ - the body force;

- $\quad g_{m n}$ - the dipolar body force;

- $\quad l$-the extrinsic equilibrated body force.

Because the tensor $e_{i j}$ is symmetric, we can deduce the following relations of symmetry:

$$
\begin{array}{r}
A_{i j m n}=A_{m n i j}=A_{i j n m}, B_{i j m n}=B_{m n i j} \\
G_{i j m n}=G_{i j n m}, F_{i j k m n}=F_{i j k n m}, C_{i j k m n r}=C_{m n r i j k}, \\
a_{i j k}=a_{j i k}, \alpha_{i j k}=\alpha_{j i k}, p_{m n}=p_{n m} .
\end{array}
$$

To build the mixed problem in our context, we will add, in addition to the basic equations above, the following initial conditions:

$$
\begin{gathered}
v_{m}(x, 0)=v_{0 m}(x), \dot{v}_{m}(x, 0)=v_{1 m}(x), \\
\phi_{j k}(x, 0)=\phi_{0 j k}(x), \dot{\phi}_{j k}(x, 0)=\phi_{1 j k}(x), \\
\varphi(x, 0)=\varphi_{0}(x), \omega_{v}(x, 0)=\omega_{0 v}(x), x \in D,
\end{gathered}
$$

and the given boundary data:

$$
\begin{array}{r}
v_{m}=\tilde{v}_{m}, \text { on } \bar{\Sigma}_{1} \times\left[0, t_{0}\right], t_{j} \equiv\left(t_{j k}+\tau_{j k}\right) n_{k}=\tilde{t}_{j}, \text { on } \Sigma_{1}^{c} \times\left[0, t_{0}\right], \\
\phi_{i j}=\tilde{\phi}_{i j}, \text { on } \bar{\Sigma}_{2} \times\left[0, t_{0}\right], m_{j k} \equiv m_{i j k} n_{i}=\tilde{m}_{j k}, \text { on } \Sigma_{2}^{c} \times\left[0, t_{0}\right], \\
\varphi=\tilde{\varphi} \text {, on } \bar{\Sigma}_{3} \times\left[0, t_{0}\right], h \equiv h_{i} n_{i}=\tilde{h}, \text { on } \Sigma_{3}^{c} \times\left[0, t_{0}\right] .
\end{array}
$$

In (11), the surfaces $\bar{\Sigma}_{1}, \bar{\Sigma}_{2}$, and $\bar{\Sigma}_{3}$ along with their complements $\Sigma_{1}^{c}, \Sigma_{2}^{c}$ and $\Sigma_{3}^{c}$, are subsets of the boundary $\partial D$, and satisfy the following two conditions:

$$
\begin{gathered}
\bar{\Sigma}_{1} \cup \Sigma_{1}^{c}=\bar{\Sigma}_{2} \cup \Sigma_{2}^{c}=\bar{\Sigma}_{3} \cup \Sigma_{3}^{c}=\partial D, \\
\Sigma_{1} \cap \Sigma_{1}^{c}=\Sigma_{2} \cap \Sigma_{2}^{c}=\Sigma_{3} \cap \Sigma_{3}^{c}=\varnothing .
\end{gathered}
$$

The functions $v_{0 m}, v_{1 m}, \phi_{0 j k}, \phi_{1 j k}, \varphi_{0} \omega_{0 v}, \tilde{v}_{m}, \tilde{t}_{m}, \tilde{\varphi}_{j k}, \tilde{m}_{j k}, \tilde{h}$, and $\tilde{\varphi}$, from the above conditions (10) and (11), are prescribed and meet sufficient conditions of regularity in their domain of definition.

We use the notation $\mathcal{P}$ for the mixed value problem in the context of the elasticity theory of media with pores and internal state variables and having a dipolar structure. consists of the Equations (5)-(7), the initial conditions (10) and the boundary conditions (11).

A state of deformation $\left(v_{m}, \phi_{j k}, \varphi, \omega_{v}\right)$ is called a solution for the mixed problem $\mathcal{P}$ if for this deformation the Equations (5)-(7) and the conditions (10) and (11) are satisfied.

\section{Basic Results}

In this section, we will address the problem of the influence that voids and internal state variables may have on the behavior of media with dipolar structure.

In order to prove the theorem of uniqueness for the solution of the previous mixed problem $\mathcal{P}$, we need some restrictions, which we are systematizing now, in order to avoid their repetition.

These constraints are as follows: 
(i) the constitutive tensors $A_{i j m n}, B_{i j m n}$ and $C_{i j k m n r}$ are positive definite:

$$
\begin{array}{r}
\int_{D} A_{i j m n} x_{i j} x_{m n} d V \geq \lambda_{1} \int_{D} x_{i j} x_{i j} d V, \forall x_{i j,} \\
\int_{D} B_{i j m n} y_{i j} y_{m n} d V \geq \lambda_{2} \int_{D} y_{i j} y_{i j} d V, \forall y_{i j}, \\
\int_{D} C_{i j k m n r} z_{i j k} z_{m n r} d v \geq \lambda_{3} \int_{D} z_{i j k} z_{i j k} d V, \forall z_{i j k},
\end{array}
$$

where $\lambda_{1}, \lambda_{2}$ and $\lambda_{3}$ are positive constants;

(ii) the density of mass $\rho$ satisfies the condition:

$$
\rho(x) \geq \rho_{0}>0, \text { on } D ;
$$

(iii) a positive constant $\lambda_{4}$ can be determined in order to be meet the following condition:

$$
I_{m n} x_{m} x_{n} \geq \lambda_{4} x_{m} x_{m}, \forall x_{m}
$$

We take into account consider two possible solutions of the above stated problem $\mathcal{P}$ :

$$
\left(v_{m}^{(\beta)}, \phi_{j k}^{(\beta)}, \varphi^{(\beta)}, \omega_{v}^{(\beta)}\right), \beta=1,2 .
$$

Of course, based on the linearity of the problem $\mathcal{P}$, the difference of its two solutions is also a solution.

However, this difference is the solution to that form of the mixed problem $\mathcal{P}$ in which the initial data, the boundary data and the loads are null.

We introduce the notations $\left(w_{m}, \psi_{j k}, \kappa, w_{v}\right)$ for the differences, namely,

$$
w_{m}=v_{m}^{(2)}-v_{m}^{(1)}, \psi_{j k}=\phi_{j k}^{(2)}-\phi_{j k}^{(1)}, \kappa=\varphi^{(2)}-\varphi^{(1)}, w_{v}=\omega_{v}^{(2)}-\omega_{v}^{(1)} .
$$

It is the clear the equivalence between the theorem of uniqueness of the solution of the problem $\mathcal{P}$ and the theorem by which it is shown that that form of the problem $\mathcal{P}$ in which the initial data, the boundary data and the loads are null, admits only the null solution.

As such we consider the problem $\mathcal{P}_{0}$ that form of the mixed problem $\mathcal{P}$ in which we have

$$
\begin{array}{r}
f_{m}=0, g_{j k}=0, l=0, \\
v_{0 m}=0, v_{1 m}=0, \phi_{0 j k}=0, \phi_{1 j k}=0, \varphi_{0}=0, \omega_{0 v}=0,
\end{array}
$$

and

$$
\tilde{v}_{m}=0, \tilde{t}_{m}=0, \tilde{\phi}_{j k}=0, \tilde{m}_{j k}=\tilde{\varphi}=0, \tilde{h}=0,
$$

and we must prove that this problem admits only the null solution, that is,

$$
v_{m}=0, \phi_{j k}=0, \varphi=0, \omega_{v}=0,
$$

in $D \times\left[0, t_{0}\right]$, provided that the hypotheses (i)-(iii) hold.

More explicitly, the problem $P_{0}$ consists of

- basic equations:

$$
\begin{array}{r}
\left(t_{m n}+\tau_{m n}\right)_{, n}=\rho \ddot{v}_{m}, \\
m_{i j k, i}+\tau_{j k}=I_{k r} \ddot{\phi}_{j r}, \\
h_{i, i}=\rho k \ddot{\varphi}, \\
\dot{\omega}_{v}=f_{v}
\end{array}
$$


- $\quad$ the initial conditions:

$$
\begin{array}{r}
v_{m}(x, 0)=0, \dot{v}_{m}(x, 0)=0, \phi_{j k}(x, 0)=0 \\
\dot{\phi}_{j k}(x, 0)=0, \varphi(x, 0)=0, \omega_{v}(x, 0)=0, x \in D
\end{array}
$$

- $\quad$ the boundary conditions

$$
\begin{array}{r}
v_{m}=0, \text { on } \bar{\Sigma}_{1} \times\left[0, t_{0}\right], t_{j} \equiv\left(t_{j k}+\tau_{j k}\right) n_{k}=0, \text { on } \Sigma_{1}^{c} \times\left[0, t_{0}\right], \\
\phi_{i j}=0, \text { on } \bar{\Sigma}_{2} \times\left[0, t_{0}\right], m_{j k} \equiv m_{i j k} n_{i}=0, \text { on } \Sigma_{2}^{c} \times\left[0, t_{0}\right], \\
\varphi=0, \text { on } \bar{\Sigma}_{3} \times\left[0, t_{0}\right], h \equiv h_{i} n_{i}=0, \text { on } \Sigma_{3}^{c} \times\left[0, t_{0}\right] .
\end{array}
$$

Obviously, when we approach the Equations (12) and the conditions (13) and (14), we will keep in mind the constitutive relations (4) and (8).

It will be easy to show that the homogeneous problem $\mathcal{P}_{0}$ admits the trivial solution if we consider the following function:

$$
y(t)=\int_{D}\left(\dot{v}_{m} \dot{v}_{m}+\dot{\phi}_{j k} \dot{\phi}_{j k}+e_{i j} e_{i j}+\varepsilon_{i j} \varepsilon_{i j}+\gamma_{i j k} \gamma_{i j k}+\varphi^{2}+\omega_{v} \omega_{v}\right) d V, t \in\left[0, t_{0}\right] .
$$

Namely, we will show that

$$
y(t)=0, \forall t \in\left[0, t_{0}\right]
$$

vanishes on $\left[0, t_{0}\right]$.

Clearly, because the function $y(t)$ a quadratic form in its variables, from relation $y(t)=$ $0, \forall t \in\left[0, t_{0}\right]$ we deduce

$$
\dot{v}_{m}=0, \dot{\phi}_{j k}=0, e_{i j}=0, \varepsilon_{i j}=0, \gamma_{i j k}=0, \varphi=0, \omega_{v}=0,
$$

so that will be easy to prove that

$$
v_{m}=0, \phi_{j k}=0, \varphi=0, \omega_{v}=0,
$$

In order to obtain these results, first we will prove some helpful estimations, contained in the following theorems.

The first auxiliary estimate is proved in the following theorem.

Theorem 1. If the ordered array $\left(v_{m}, \phi_{j k}, \varphi, \omega_{v}\right)$ is an arbitrary solution for the problem $\mathcal{P}_{0}$, then takes place the following equality:

$$
\begin{array}{r}
\int_{D}\left(A_{i j m n} e_{i j} e_{m n}+2 G_{i j m n} e_{i j} \varepsilon_{m n}+2 F_{m n r i j} e_{i j} \gamma_{m n r}+\right. \\
B_{i j m n} \varepsilon_{i j} \varepsilon_{m n}+C_{i j s m n r} \gamma_{i j s} \gamma_{m n r}+2 D_{i j m n r} \varepsilon_{i j} \gamma_{m n r}+2 \alpha_{i j v} e_{i j} \omega_{v}+ \\
\left.2 \beta_{i j v} \varepsilon_{i j} \omega_{v}+2 \delta_{i j r v} \gamma_{i j r} \omega_{v}+\rho \dot{v}_{m} \dot{v}_{m}+I_{k r} \dot{\phi}_{j r} \dot{\phi}_{j k}\right) d V= \\
2 \int_{0}^{t} \int_{D}\left(\alpha_{i j v} e_{i j}+\beta_{i j v} \varepsilon_{i j}+\delta_{i j r v} \gamma_{i j r}\right) \dot{\omega}_{v} d V d s
\end{array}
$$


Proof. If we take into account the symmetry relations (9) and the constitutive Equation (4), we are led to the equality

$$
\begin{array}{r}
t_{i j} \dot{j}_{j, i}+\tau_{i j} \dot{\varepsilon}_{i j}+m_{i j s} \dot{\gamma}_{i j s}= \\
\frac{1}{2} \frac{\partial}{\partial t}\left[A_{i j m n} e_{i j} e_{m n}+2 G_{m n i j} e_{i j} \varepsilon_{m n}+2 F_{m n r i j} e_{i j} \gamma_{m n r}+\right. \\
B_{i j m n} \varepsilon_{i j} \varepsilon_{m n}+C_{i j s m n r} \gamma_{i j s} \gamma_{m n r}+2 D_{i j m n r} \gamma_{i j} \gamma_{m n r}+ \\
\left.2\left(\alpha_{i j v} e_{i j}+\beta_{i j v} \varepsilon_{i j}+\delta_{i j s v} \gamma_{i j s}\right) \omega_{v}\right]- \\
\alpha_{i j v}\left(e_{i j}+\beta_{i j v} \varepsilon_{i j}+\delta_{i j s v} \gamma_{i j s}\right) \dot{\omega}_{v} .
\end{array}
$$

Now, we will consider the Equations (12) in order to obtain

$$
t_{i j} \dot{v}_{j, i}+\tau_{i j} \dot{\varepsilon}_{i j}+m_{i j s} \dot{\gamma}_{i j s}=\left[\left(t_{i j}+\tau_{i j}\right) \dot{v}_{i}+m_{i j s} \dot{\phi}_{j s}\right]_{, i}-\frac{1}{2} \frac{\partial}{\partial t}\left(\rho \dot{v}_{i} \dot{v}_{i}+I_{k r} \dot{\phi}_{j r} \dot{\phi}_{j k}\right)
$$

From the equalities (16) and (17), we have

$$
\begin{array}{r}
\frac{1}{2} \frac{\partial}{\partial t}\left[A_{i j m n} e_{i j} e_{m n}+2 G_{m n i j} e_{i j} \varepsilon_{m n}+2 F_{m n r i j} e_{i j} \gamma_{m n r}+\right. \\
B_{i j m n} \varepsilon_{i j} \varepsilon_{m n}+2 D_{i j m n r} \varepsilon_{i j} \gamma_{m n r}+C_{i j s m n r} \gamma_{i j s} \gamma_{m n r}+ \\
2\left(\alpha_{i j v} e_{i j}+\beta_{i j v} \varepsilon_{i j}+\delta_{i j s v} \gamma_{i j s}\right) \omega_{v}+ \\
\left.\rho \dot{v}_{i} \dot{v}_{i}+I_{k r} \dot{\phi}_{j r} \dot{\phi}_{j k}\right]= \\
{\left[\left(t_{i j}+\tau_{i j}\right) \dot{v}_{i}+m_{i j s} \dot{\phi}_{j s}\right]_{, i}+} \\
\left(\alpha_{i j v} e_{i j}+\beta_{i j v} \varepsilon_{i j}+\delta_{i j s v} \gamma_{i j s}\right) \dot{\omega}_{v} .
\end{array}
$$

We will now integrate the equality (18) on the domain $D$ and apply the divergence theorem. If we consider the boundary conditions (14), we come to the conclusion that

$$
\begin{array}{r}
\frac{1}{2} \frac{\partial}{\partial t} \int_{D}\left(A_{i j m n} e_{i j} e_{m n}+2 G_{m n i j} e_{i j} \varepsilon_{m n}+2 F_{m n r i j} e_{i j} \gamma_{m n r}++\right. \\
B_{i j m n} \varepsilon_{i j} \varepsilon_{m n}+2 D_{i j m n r} \gamma_{i j} \gamma_{m n r}+C_{i j s m n r} \gamma_{i j s} \gamma_{m n r}+ \\
2\left(\alpha_{i j v} e_{i j}+\beta_{i j v} \varepsilon_{i j}+\delta_{i j s v} \gamma_{i j s}\right) \omega_{v}+ \\
\left.\rho \dot{v}_{i} \dot{v}_{i}+I_{k r} \dot{\phi}_{j r} \dot{\phi}_{j k}\right] d V= \\
\int_{D}\left(\alpha_{i j v} e_{i j}+\beta_{i j v} \varepsilon_{i j}+\delta_{i j s v} \gamma_{i j s}\right) \dot{\omega}_{v} d V .
\end{array}
$$

It remains to integrate from 0 to $t$ the equality (19) and to take into account the initial conditions from (13). Therefore, we reach the equality (15) from the enounce of the theorem, which concludes the proof.

In the following theorem, we will prove another auxiliary estimate.

Theorem 2. Let us consider $\left(v_{m}, \phi_{j k}, \varphi, \omega_{v}\right)$ a solution of the problem $P_{0}$. Then, a positive constant $m_{1}$ can be found so that the following inequality is satisfied:

$$
\int_{D}\left(\alpha_{i j v} e_{i j}+\beta_{i j v} \varepsilon_{i j}+\delta_{i j s v} \gamma_{i j s}\right) \dot{\omega}_{\nu} d V \leq m_{1} \int_{D}\left(e_{i j} e_{i j}+\varepsilon_{i j} \varepsilon_{i j}+\gamma_{i j s} \gamma_{i j s}+\varphi_{, m} \varphi_{, m}+\omega_{\nu} \omega_{v}\right) d V .
$$


Proof. With the help of Equations (7), (8), and (4), we obtain

$$
\begin{array}{r}
\int_{D}\left(\alpha_{i j v} e_{i j}+\beta_{i j v} \varepsilon_{i j}+\delta_{i j s v} \gamma_{i j s}\right) \dot{\omega}_{v} d V= \\
\int_{D}\left[( \alpha _ { i j v } e _ { i j } + \beta _ { i j v } \varepsilon _ { i j } + \delta _ { i j s v } \gamma _ { i j s } ) \left(g_{i j v} e_{i j}+\right.\right. \\
\left.h_{i j v} \varepsilon_{i j}+l_{i j s v} \gamma_{i j s}+q_{v \beta} \omega_{\beta}\right)- \\
\left.\left(a_{i j k} e_{j k}+b_{i j} \varepsilon_{j k}+c_{i j s m} \gamma_{j s m}+f_{i v} \omega_{v}\right) \varphi_{, i}\right] d V= \\
\int_{D}\left(\mathcal{A}_{i j m n} e_{i j} e_{m n}+\mathcal{G}_{i j m n} e_{i j} \varepsilon_{m n}+\mathcal{F}_{i j m n r} e_{i j} \gamma_{m n r}+\right. \\
\mathcal{B}_{i j m n} \varepsilon_{i j} \varepsilon_{m n}+\mathcal{D}_{i j m n r} \varepsilon_{i j} \gamma_{m n r}+\mathcal{C}_{i j s m n r} \gamma_{i j s} \gamma_{m n r}+ \\
\mathcal{A}_{i j v} e_{i j} \omega_{v}+\mathcal{A}_{i j k} e_{i j} \varphi_{, k}+\mathcal{B}_{i j v} \varepsilon_{i j} \omega_{v}+\mathcal{B}_{i j k} \varepsilon_{i j} \varphi_{, k}+ \\
\left.\mathcal{C}_{i j s v} \gamma_{i j s} \omega_{v}+\mathcal{C}_{i j s m} \gamma_{i j s} \varphi_{, m}+\mathcal{Q}_{i v} \omega_{v} \varphi_{, i}\right) d V,
\end{array}
$$

where we have used the following notations:

$$
\begin{array}{r}
\mathcal{A}_{i j m n}=\frac{1}{2}\left(\alpha_{i j v} g_{m n v}+\alpha_{m n v} g_{i j v}\right), \mathcal{B}_{i j m n}=\frac{1}{2}\left(\beta_{i j v} h_{m n v}+\beta_{m n v} h_{i j v}\right), \\
\mathcal{C}_{i j s m n r}=\frac{1}{2}\left(F_{i j k v} l_{m n r v}+F_{m n r v} l_{i j k v}\right), \mathcal{G}_{i j m n}=\alpha_{i j v} h_{m n v}+\beta_{m n v} g_{i j v} \\
\mathcal{D}_{i j m n r}=\beta_{i j v} l_{m n r v}+\delta_{m n r v} h_{i j v}, \mathcal{F}_{i j m n r}=\alpha_{i j v} l_{m n r v}+\delta_{m n r v} g_{i j v} \\
\mathcal{A}_{i j v}=\alpha_{i j \beta} q_{\beta v}, \mathcal{A}_{i j k}=\alpha_{i j v} r_{k v}, \mathcal{B}_{i j v}=\beta_{i j \alpha} q_{\alpha v}, \mathcal{B}_{i j k}=\beta_{i j v} r_{k v} \\
\mathcal{C}_{i j k v}=\delta_{i j k \alpha} q_{\alpha v}, \mathcal{C}_{i j k s}=\delta_{i j s v} r_{k v}, \mathcal{Q}_{i v}=-f_{i v} .
\end{array}
$$

Now, for the terms in the last integral of the relation (22), we will use the arithmeticgeometric mean inequality in the form

$$
2 a b \leq\left(\frac{a^{2}}{p^{2}}+b^{2} p^{2}\right),
$$

and, also, the Schwarz's inequality, so that we obtain the following inequality:

$$
\begin{array}{r}
\int_{D}\left(\alpha_{i j v} e_{i j}+\beta_{i j v} \varepsilon_{i j}+\delta_{i j s v} \gamma_{i j s}\right) \dot{\omega}_{v} d V \leq \\
\left(K_{1}^{2}+\frac{K_{2}^{2}}{p_{1}^{2}}+\frac{K_{3}^{2}}{p_{2}^{2}}+\frac{K_{4}^{2}}{p_{4}^{2}}+\frac{K_{5}^{2}}{p_{5}^{2}}\right) \int_{D} e_{i j} e_{i j} d V+ \\
\left(K_{6}^{2}+\frac{K_{2}^{2}}{p_{3}^{2}}+K_{2}^{2}+\frac{K_{7}^{2}}{p_{6}^{2}}+\frac{K_{8}^{2}}{p_{7}^{2}}\right) \int_{D} \varepsilon_{i j} \varepsilon_{i j} d V+ \\
\left(K_{9}^{2}+K_{3}^{2}+K_{2}^{2}+\frac{K_{10}^{2}}{p_{8}^{2}}+K_{11}^{2}\right) \int_{D} \gamma_{i j s} \gamma_{i j s} d V+ \\
\left(K_{4}^{2}+K_{6}^{2}+K_{10}^{2}+\frac{K_{12}^{2}}{p_{9}^{2}}\right) \int_{D} \omega_{v} \omega_{v} d V+ \\
\left(K_{5}^{2}+K_{7}^{2}+K_{11}^{2}+K_{12}^{2}\right) \int_{B} \varphi_{, m} \varphi_{, m} d V,
\end{array}
$$


where the constants $p_{i}, i=1,2, \ldots, 9$ are determined by using (23). The notations used in the inequality (24) have the following significance:

$$
\begin{array}{r}
K_{1}^{2}=2 \max \left(\mathcal{A}_{i j m n} \mathcal{A}_{i j m n}\right)\left(x_{s}\right), K_{2}^{2}=\max \left(\mathcal{G}_{i j m n} \mathcal{G}_{i j m n}\right)\left(x_{s}\right), \\
K_{3}^{2}=\max \left(\mathcal{F}_{i j m n r} \mathcal{F}_{i j m n r}\right)\left(x_{s}\right), K_{4}^{2}=\max \left(\mathcal{A}_{i j v} \mathcal{A}_{i j v}\right)\left(x_{s}\right), \\
K_{5}^{2}=\max \left(\mathcal{A}_{i j k} \mathcal{A}_{i j k}\right)\left(x_{s}\right), K_{6}^{2}=2 \max \left(\mathcal{B}_{i j m n} \mathcal{B}_{i j m n}\right)\left(x_{s}\right), \\
K_{7}^{2}=\max \left(\mathcal{B}_{i j v} \mathcal{B}_{i j v}\right)\left(x_{s}\right), K_{8}^{2}=\max \left(\mathcal{B}_{i j k} \mathcal{B}_{i j k}\right)\left(x_{s}\right), \\
K_{9}^{2}=2 \max \left(\mathcal{C}_{i j k m n r} \mathcal{C}_{i j k m n r}\right)\left(x_{s}\right), K_{10}^{2}=\max \left(\mathcal{C}_{i j k v} \mathcal{C}_{i j k v}\right)\left(x_{s}\right), \\
K_{11}^{2}=\max \left(\mathcal{C}_{i j k r} \mathcal{A}_{i j k r}\right)\left(x_{s}\right), K_{12}^{2}=\max \left(\mathcal{Q}_{i v} \mathcal{Q}_{i v}\right)\left(x_{s}\right) .
\end{array}
$$

Now, we can choose the constant $m_{1}$ as follows:

$$
\begin{array}{r}
m_{1}=\frac{1}{2} \max \left\{K_{1}^{2}+\frac{K_{2}^{2}}{p_{1}^{2}}+\frac{K_{3}^{2}}{p_{2}^{2}}+\frac{K_{4}^{2}}{p_{4}^{2}}+\frac{K_{5}^{2}}{p_{5}^{2}},\right. \\
K_{6}^{2}+\frac{K_{2}^{2}}{p_{3}^{2}}+K_{2}^{2}+\frac{K_{7}^{2}}{p_{6}^{2}}+\frac{K_{8}^{2}}{p_{7}^{2}}, \\
K_{9}^{2}+K_{3}^{2}+K_{2}^{2}+\frac{K_{10}^{2}}{p_{8}^{2}}+K_{11}^{2}, \\
K_{4}^{2}+K_{6}^{2}+K_{10}^{2}+\frac{K_{12}^{2}}{p_{9}^{2}}, \\
\left.K_{5}^{2}+K_{7}^{2}+K_{11}^{2}+K_{12}^{2}\right\},
\end{array}
$$

so that from (24) we obtain the estimation (21) and in this way the proof of Theorem 2 is completed.

The last auxiliary estimate will be proved in the next theorem.

Theorem 3. Suppose that the assumptions (i)-(iii) are satisfied and consider a solution $\left(v_{m}, \phi_{j k}, \varphi, \omega_{v}\right)$ for the problem $\mathcal{P}_{0}$. Then, it can determine a constant $m_{2}>0$ so that the next inequality occurs:

$$
\begin{array}{r}
\int_{D}\left(\dot{v}_{m} \dot{v}_{m}+\dot{\phi}_{k j} \dot{\phi}_{j k}+e_{i j} e_{i j}+\varepsilon_{i j} \varepsilon_{i j}+\gamma_{i j k} \gamma_{i j k}+\varphi^{2}+\omega_{v} \omega_{v}\right) d V \leq \\
m_{2} \int_{0}^{t} \int_{D}\left(\dot{v}_{m} \dot{v}_{m}+\dot{\phi}_{j k} \dot{\phi}_{j k}+e_{i j} e_{i j}+\varepsilon_{i j} \varepsilon_{i j}+\gamma_{i j k} \gamma_{i j k}+\varphi^{2}+\omega_{v} \omega_{v}\right) d V d s,
\end{array}
$$

which takes place for any $t \in\left[0, t_{0}\right]$.

Proof. First, we use the notation

$$
m_{0}=\min \left\{\lambda_{1}, \lambda_{2}, \lambda_{3}, \lambda_{4}\right\},
$$


so that, with the help of hypotheses (i)-(iii), we deduce

$$
\begin{array}{r}
m_{0} \int_{D}\left(\dot{v}_{m} \dot{v}_{m}+\dot{\phi}_{j k} \dot{\phi}_{j k}+e_{i j} e_{i j}+\varepsilon_{i j} \varepsilon_{i j}+\gamma_{i j k} \gamma_{i j k}+\varphi^{2}+\omega_{v} \omega_{v}\right) d V \leq \\
\int_{D}\left(A_{i j m n} e_{i j} e_{m n}+2 G_{m n i j} e_{i j} \varepsilon_{m n}+2 F_{m n r i j} e_{i j} \gamma_{m n r}+\right. \\
B_{i j m n} \varepsilon_{i j} \varepsilon_{m n}+2 D_{i j m n r} \gamma_{i j} \gamma_{m n r}+C_{i j s m n r} \gamma_{i j s} \gamma_{m n r}+ \\
2\left(\alpha_{i j v} e_{i j}+\beta_{i j v} \varepsilon_{i j} \delta_{i j s v} \gamma_{i j s}\right) \omega_{v}+ \\
\left.\rho \dot{v}_{m} \dot{v}_{m}+I_{k r} \dot{\phi}_{j r} \dot{\phi}_{j k}+\rho k \varphi^{2}\right) d V .
\end{array}
$$

Let us introduce the notations

$$
\begin{array}{r}
M_{1}^{2}=\max \left(\alpha_{i j v} \alpha_{i j v}\right)\left(x_{s}\right), M_{2}^{2}=\max \left(\beta_{i j v} \beta_{i j v}\right)\left(x_{s}\right), \\
M_{3}^{2}=\max \left(\delta_{i j k v} \delta_{i j k v}\right)\left(x_{s}\right), M_{4}^{2}=\max \left(G_{m n i j} G_{m n i j}\right)\left(x_{s}\right), \\
M_{5}^{2}=\max \left(D_{i j m n r} D_{i j m n r}\right)\left(x_{s}\right), M_{6}^{2}=\max \left(F_{m n r i j} F_{m n r i j}\right)\left(x_{s}\right),
\end{array}
$$

where $\left(x_{s}\right) \in \bar{B}$.

Next, as in the proof of Theorem 2, for the terms in the integral from the left-hand side of the inequality (27) we use the arithmetic-geometric mean inequality in the form (23) and, also, the Schwarz's inequality. Therefore, if we take into account the notations (28), from (27) we are lead to the inequality

$$
\begin{array}{r}
m_{0} \int_{B}\left(\dot{v}_{m} \dot{v}_{m}+\dot{\phi}_{j k} \dot{\phi}_{j k}+e_{i j} e_{i j}+\varepsilon_{i j} \varepsilon_{i j}+\gamma_{i j k} \gamma_{i j k}+\varphi^{2}\right) d V \leq \\
\left(p_{6}^{2}+M_{4}^{2}+M_{5}^{2}\right) \int_{D} e_{i j} e_{i j} d V+\left(p_{7}^{2}+M_{6}^{2}+2\right) \int_{D} \varepsilon_{i j} \varepsilon_{i j} d V+ \\
\left(p_{8}^{2}+3\right) \int_{D} \gamma_{i j k} \gamma_{i j k} d V+\left(\frac{M_{1}^{2}}{p_{6}^{2}}+\frac{M_{2}^{2}}{p_{7}^{2}}+\frac{M_{3}^{2}}{p_{8}^{2}}\right) \int_{D} \omega_{v} \omega_{v} d V+ \\
m_{1} \int_{0}^{t} \int_{D}\left(e_{i j} e_{i j}+\varepsilon_{i j} \varepsilon_{i j}+\gamma_{i j k} \gamma_{i j k}+\varphi^{2}+\omega_{v} \omega_{v}\right) d V d s,
\end{array}
$$

and this inequality takes place for any $t \in\left[0, t_{0}\right]$.

Now, it is not very difficult to follow the following estimation:

$$
\begin{aligned}
\int_{D} \omega_{\nu} \omega_{\nu} d V= & \int_{0}^{t} \frac{d}{d \tau}\left(\int_{B} \omega_{\nu} \omega_{\nu} d V\right) d \tau=2 \int_{0}^{t}\left(\int_{D} \dot{\omega}_{\nu} \omega_{\nu} d V\right) d \tau= \\
& 2 \int_{0}^{t} \int_{D}\left(g_{i j v} e_{i j}+h_{i j v} \varepsilon_{i j}+l_{i j k v} \gamma_{i j k}+q_{v \beta} \omega_{\beta}\right) \omega_{\nu} d V d \tau,
\end{aligned}
$$

were we used the initial conditions (13) and the constitutive relation (8).

For terms below the integral on the left side of equality (30) we use, again, the inequality of Schwarz so that the inequality (30) receives the form:

$$
\begin{array}{r}
\int_{D} \omega_{\nu} \omega_{v} d V \leq \int_{0}^{t} \int_{D} \varphi_{, i} \varphi_{, i} d V d \tau+ \\
\left(N_{4}^{2}+3\right) \int_{0}^{t} \int_{B} \omega_{\nu} \omega_{v} d V d \tau+ \\
N_{1}^{2} \int_{0}^{t} \int_{D} e_{i j} e_{i j} d V d \tau+N_{2}^{2} \int_{0}^{t} \int_{D} \varepsilon_{i j} \varepsilon_{i j} d V d \tau+ \\
N_{3}^{2} \int_{0}^{t} \int_{D} \gamma_{i j k} \gamma_{i j k} d V d \tau+\int_{0}^{t} \int_{D} \varphi^{2} d V d \tau,
\end{array}
$$

for any $t \in\left[0, t_{0}\right]$. 
Furthermore, in the above inequality we considered the following notations:

$$
\begin{gathered}
N_{1}^{2}=\max \left(g_{i j v} g_{i j v}\right)\left(x_{s}\right), N_{2}^{2}=\max \left(h_{i j v} h_{i j v}\right)\left(x_{s}\right), \\
N_{3}^{2}=\max \left(l_{i j k v} l_{i j k v}\right)\left(x_{s}\right), N_{4}^{2}=\max \left(q_{i v} q_{i v}\right)\left(x_{s}\right),
\end{gathered}
$$

where $\left(x_{s}\right) \in \bar{B}$.

The inequality (31) can be restated in the form

$$
\begin{array}{r}
\int_{B} \omega_{v} \omega_{v} d V \leq \int_{0}^{t} \int_{D} \varphi_{, i} \varphi_{, i} d V d \tau+ \\
m_{3} \int_{0}^{t} \int_{D}\left(e_{i j} e_{i j}+\varepsilon_{i j} \varepsilon_{i j}+\gamma_{i j k} \gamma_{i j k}+\varphi^{2}+\omega_{v} \omega_{v}\right) d V d \tau,
\end{array}
$$

where $m_{3}$ is positive constant defined by

$$
m_{3}=\max \left\{N_{1}^{2}, N_{2}^{2}, N_{2}^{2}, Q_{3}^{2}, Q_{4}^{2}+3,1\right\} .
$$

From (29) and (33), we obtain

$$
\begin{array}{r}
m_{0} \int_{D}\left(\dot{v}_{m} \dot{v}_{m}+\dot{\phi}_{j k} \dot{\phi}_{j k}+\varphi^{2}\right) d V+\left[m_{0}-\left(p_{6}^{2}+M_{4}^{2}+M_{5}^{2}\right)\right] \int_{D} e_{i j} e_{i j} d V+ \\
\left(m_{0}-p_{7}^{2}-M_{6}^{2}-2\right) \int_{D} \varepsilon_{i j} \varepsilon_{i j} d V+\left(m_{0}-p_{8}^{2}-3\right) \int_{D} \gamma_{i j k} \gamma_{i j k} d V+ \\
\left(p_{10}^{2}-\frac{M_{1}^{2}}{p_{6}^{2}}-\frac{M_{2}^{2}}{p_{7}^{2}}-\frac{M_{3}^{2}}{p_{8}^{2}}\right) \int_{D} \omega_{v} \omega_{v} d V \leq\left(m_{1}-p_{10}^{2}\right) \int_{0}^{t} \int_{B} \varphi_{, i} \varphi_{, i} d V d s+ \\
\left(m_{1}+m_{3} p_{10}^{2}\right) \int_{0}^{t} \int_{B}\left(e_{i j} e_{i j}+\varepsilon_{i j} \varepsilon_{i j}+\gamma_{i j k} \gamma_{i j k}+\varphi^{2}+\omega_{v} \omega_{v}\right) d V d s .
\end{array}
$$

As the constants $p_{6}-p_{10}$ and $N_{1}-N_{6}$ are arbitrary, we can determine other constants $k_{5}-k_{9}$ and $Y$, defined as follows:

$$
\begin{array}{r}
k_{5}=m_{0}-p_{6}^{2}-M_{4}^{2}-M_{5}^{2}, k_{6}=m_{0}-p_{7}^{2}-M_{6}^{2}-2, \\
k_{7}=m_{0}-p_{8}^{2}-3, k_{8}=p_{10}^{2}-\frac{M_{1}^{2}}{p_{6}^{2}}-\frac{M_{2}^{2}}{p_{7}^{2}}-\frac{M_{3}^{2}}{p_{8}^{2}} \\
k_{9}=m_{1}-p_{10}^{2}
\end{array}
$$

so that $k_{i}>0, i=\overline{5,9}$.

Furthermore, we can define $k_{10}$ by

$$
k_{10}=\min \left\{m_{0}, k_{5}, k_{6}, k_{7}, k_{8}\right\} .
$$

Withe these considerations the inequality (34) receives the form

$$
\begin{array}{r}
\left(m_{1}+m_{3} p_{10}^{2}\right) \int_{0}^{t} \int_{D}\left(e_{i j} e_{i j}+\varepsilon_{i j} \varepsilon_{i j}+\gamma_{i j k} \gamma_{i j k}+\varphi^{2}+\omega_{v} \omega_{v}\right) d V d s \geq \\
m_{0} \int_{D}\left(\dot{v}_{m} \dot{v}_{m}+\dot{\phi}_{j k} \dot{\phi}_{j k}+\varphi^{2}\right) d V+k_{5} \int_{D} e_{i j} e_{i j} d V+k_{6} \int_{D} \varepsilon_{i j} \varepsilon_{i j} d V+ \\
k_{7} \int_{D} \gamma_{i j k} \gamma_{i j k} d V+k_{8} \int_{D} \omega_{v} \omega_{v} d V+k_{9} \int_{D} \varphi_{, i} \varphi_{, i} d V \geq \\
k_{10} \int_{D}\left(\dot{v}_{m} \dot{v}_{m}+\dot{\phi}_{j k} \dot{\phi}_{j k}+e_{i j} e_{i j}+\varepsilon_{i j} \varepsilon_{i j}+\gamma_{i j k} \gamma_{i j k}+\varphi^{2}+\omega_{v} \omega_{v}\right) d V .
\end{array}
$$


It is not very difficult to prove that

$$
\begin{array}{r}
\int_{0}^{t} \int_{D}\left(\dot{v}_{m} \dot{v}_{m}+\dot{\phi}_{j k} \dot{\phi}_{j k}+e_{i j} e_{i j}+\varepsilon_{i j} \varepsilon_{i j}+\gamma_{i j k} \gamma_{i j k}+\varphi^{2}+\omega_{v} \omega_{v}\right) d V d \tau \geq \\
\int_{0}^{t} \int_{D}\left(e_{i j} e_{i j}+\varepsilon_{i j} \varepsilon_{i j}+\gamma_{i j k} \gamma_{i j k}+\varphi^{2}+\omega_{\nu} \omega_{v}\right) d V d \tau
\end{array}
$$

Now, we define $m_{2}$ by

$$
m_{2}=\frac{\left(m_{1}+m_{3} p_{10}^{2}\right)}{k_{10}} .
$$

Finally, using this form of $m_{2}$ and taking into account the estimates (35) and (36) we find the estimate (26) and Theorem 3 is proved.

Our main result will be obtained by taking into account the estimates of Theorem 1 , Theorems 2 and 3. Therefore, we will prove the uniqueness of mixed initial-boundary value problem $\mathcal{P}$.

Theorem 4. We assume that the conditions (i)-(iii) are met. Then, the above problem $\mathcal{P}$ defined by the Equations (5) and (6) with the initial data (10) and the data to the limit (11) cannot admit more than one solution.

Proof. The reduction to the absurd method will be used. We assume that, on the contrary, the $\mathcal{P}$ problem admits at least two solutions. Assume that the problem admits two solutions. Due to the linearity of the problem, the difference of the two solutions is also a solution. More exactly, this difference of the two arbitrary solutions is a solution for the homogeneous problem $\mathcal{P}_{0}$.

As mentioned and argued earlier, we will reduce the demonstration to look like the bilinear form $y(t)$ defined by

$$
y(t)=\int_{D}\left(\dot{v}_{m} \dot{v}_{m}+\dot{\phi}_{j k} \dot{\phi}_{j k}+e_{i j} e_{i j}+\varepsilon_{i j} \varepsilon_{i j}+\gamma_{i j k} \gamma_{i j k}+\varphi^{2}+\omega_{v} \omega_{v}\right) d V,
$$

vanishes on $\left[0, t_{0}\right]$.

However, this statement is obtained immediately because the Gronwall's inequality and the inequality (26) lead to the conclusion that ensure that $y(t)=0$ for any $t \in\left[0, t_{0}\right]$ and the proof of Theorem 4 is completed.

\section{Conclusions}

It can be argued that neither the voids nor the internal state variables influence the qualitative aspects regarding the mixed problem constituted in this context, namely, the existence of the solution, the uniqueness of the solution, or the stability of the solution. In the present study, we argue one of these statements: the uniqueness of the solution.

In a later study, we can demonstrate that neither the presence of voids nor internal state variables influences the existence of the solution of the mentioned problem. We also suspect that the continuous dependence of the solutions in relation to the initial data or to the boundary data or even in relation to the loads are not affected by the consideration of voids and internal state variables.

In this paper, we proved that the existence of the pores and of variables of internal state type does not change the uniqueness result regarding the solution for the general formulated problem in the context of elastic bodies with voids, internal state variables, and dipolar structure. 
Author Contributions: Conceptualization: M.M. and S.V.; methodology: M.M., S.V., I.F. and G.P.; validation: M.M., S.V., I.F. and G.P.; formal analysis: M.M., S.V., I.F. and G.P.; investigation: I.F. and G.P.; resources: M.M.; data curation: M.M., S.V., I.F. and G.P.; writing—original draft preparation: M.M. and S.V. writing—review and editing: M.M. and S.V.; visualization: M.M., S.V., I.F. and G.P.; supervision: M.M., S.V., I.F. and G.P.; project administration: M.M. All authors have read and agreed to the published version of the manuscript.

Funding: This research received no external funding.

Institutional Review Board Statement: Not applicable.

Informed Consent Statement: Not applicable.

Data Availability Statement: Not applicable.

Conflicts of Interest: The authors declare no conflict of interest.

\section{References}

1. Nunziato, J.W.; Cowin, S.C. A nonlinear theory of materials with voids. Arch. Rat. Mech. Anal. 1979, 72, 175-201. [CrossRef]

2. Cowin, S.C.; Nunziato, J.W. Linear elastic materials with voids. J. Elast. 1983, 13, 125-147. [CrossRef]

3. Goodman, M.A.; Cowin, S.C. A continuum theory of granular material. Arch. Rat. Mech. Anal. 1971, 44, 249-266. [CrossRef]

4. Iesan, D. A theory of thermoelastic material with voids. Acta Mech. 1986, 60, 67-89. [CrossRef]

5. Marin, M.; Agarwal, R.P.; Mahmoud, S.R. Modeling a microstretch thermoelastic body with two temperature. Abstr. Appl. Anal. 2013, 2013, 583464. [CrossRef]

6. Marin, M.; Stan, G. Weak solutions in Elasticity of dipolar bodies with stretch. Carpathian J. Math. 2013, 29, 33-40. [CrossRef]

7. Eringen, A.C. Theory of micromorphic materials with memory. Int. J. Eng. Sci. 1972, 10, 623-641. [CrossRef]

8. Eringen, A.C. Theory of thermo-microstretch elastic solids. Int. J. Eng. Sci. 1990, 28, 1291-1301. [CrossRef]

9. Mindlin, R.D. Micro-structure in linear elasticity. Arch. Ration. Mech. Anal. 1964, 16, 51-78. [CrossRef]

10. Green, A.E.; Rivlin, R.S. Multipolar continuum mechanics. Arch. Ration. Mech. Anal. 1964, 17, 113-147. [CrossRef]

11. Fried, E.; Gurtin, M.E. Thermomechanics of the interface between a body and its environment. Contin. Mech. Thermodyn. 2007, 19, 253-271. [CrossRef]

12. Marin, M. A domain of influence theorem for microstretch elastic materials. Nonlinear Anal. Real World Appl. 2010, 11, 3446-3452. [CrossRef]

13. Groza, G.; Mitu, A.M.; Pop, N.; Sireteanu, T. Transverse vibrations analysis of a beam with degrading hysteretic behavior by using Euler-Bernoulli beam model. Analele St. Univ. "Ovidius" Constanta Ser. Mat. 2018, 26, 125-139. [CrossRef]

14. Zhang, L.; Bhatti, M. M.; Marin, M.; Mekheimer, K.S. Entropy analysis on the blood flow through anisotropically tapered arteries filled with magnetic zinc-oxide ( $\mathrm{ZnO}$ ) nanoparticles. Entropy 2020, 22, 1070. [CrossRef] [PubMed]

15. Marin, M.; Othman, M.I.A.; Seadawy, A.R.; Carstea, C. A domain of influence in the Moore-Gibson-Thompson theory of dipolar bodies. J. Taibah Univ. Sci. 2020, 14, 653-660. [CrossRef]

16. Abbas, I.A.; Marin, M. Analytical Solutions of a Two-Dimensional Generalized Thermoelastic Diffusions Problem Due to Laser Pulse. Iran. J. Sci. Technol.-Trans. Mech. Eng. 2018, 42, 57-71. [CrossRef]

17. Vlase, S.; Teodorescu, P.P. Elasto-dynamics of a solid with a general "rigid" motion using fem model; Part I. Theoretical approach. Rom. J. Phys. 2013, 58, 872-881.

18. Vlase, S. A method of eliminating lagrangian multipliers from the equation of motion of interconnected mechanical systems. $J$. Appl. Mech. 1987, 54, 235-237. [CrossRef]

19. Chirila, A.; Marin, M.; Montanaro, A. On adaptive thermo-electro-elasticity within a Green-Naghdi type II or III theory. Contin. Mech. Thermodyn. 2019, 31, 1453-1475. [CrossRef]

20. Groza, G.; Pop, N. Approximate solution of multipoint boundary value problems for linear differential equations by polinomial functions. J. Differ. Equ. Appl. 2008, 14, 1289-1309. [CrossRef]

21. Marin, M. Some estimates on vibrations in thermoelasticity of dipolar bodies. J. Vib. Control 2020, 16, 33-47. [CrossRef]

22. Marin, M. An evolutionary equation in thermoelasticity of dipolar bodies. J. Math. Phys. 1999, 40, 1391-1399. [CrossRef]

23. Chirita, S. On the linear theory of thermo-viscoelastic materials with internal state variables. Arch. Mech. 1982, 33, 455-464.

24. Nachlinger, R.R.; Nunziato, J.W. Wave propagation and uniqueness theorem for elastic materials with ISV. Int. J. Engng. Sci. 1976, 14, 31-38. [CrossRef]

25. Sherburn, J.A.; Horstemeyer, M.F.; Bammann, D.J.; Baumgardner, J.R. Application of the Bammann inelasticity internal state variable constitutive model to geological materials. Geophys. J. Int. 2011, 184, 1023-1036. [CrossRef]

26. Wei, C.; Dewoolkar, M.M. Formulation of capillary hysteresis with internal state variables. Water Resour. Res. 2006, 42 , W07405 [CrossRef]

27. Solanki, K.N.; Bammann, D.J. A thermodynamic framework for a gradient theory of continuum damage. Acta Mech. 2010, 213, 27-38. [CrossRef] 
28. Anand, L.; Gurtin, M.E. A theory of amorphous solids undergoing large deformations. Int. J. Solids Struct. 2003, 40, 1465-1487. [CrossRef]

29. Bouvard, J.L.; Ward, D.K.; Hossain, D.; Marin, E.B.; Bammann, D.J.; Horstemeyer, M.F. A general inelastic internal state variable model for amorphous glassy polymers. Acta Mech. 2010, 213, 71-96. [CrossRef]

30. Craciun, E.M.; Soos, E. Anti-plane states in an anisotropic elastic body containing an elliptical hole. Math. Mech. Solids 2006, 11, 459-466. [CrossRef]

31. Jafari, M.; Hoseyni, S.A.M.; Altenbach, H.; Craciun, E.M. Optimum design of infinite perforated orthotropic and isotropic plates. Mathematics 2020, 8, 569. [CrossRef] 